\title{
TRIM37 promotes cell invasion and metastasis by regulating SIPI-mediated epithelial-mesenchymal transition in gastric cancer
}

This article was published in the following Dove Press journal:

OncoTargets and Therapy

\section{Dehu Chen \\ Xiaolan You \\ Yan Pan \\ Qinghong Liu \\ Gan Cao}

Department of General Surgery, Taizhou People's Hospital, The Fifth Affiliated Hospital of Nantong University, Taizhou, Jiangsu Province, People's Republic of China
Correspondence: Gan Cao Department of General Surgery, Taizhou People's Hospital, The Fifth Affiliated Hospital of Nantong University, No 366 Taihu Road, Taizhou 225300, jiangsu Province, People's Republic of China

Tel +86 I39526I 6I08

Email dr_ctzrmyy13420@163.com
Background: Tripartite motif containing 37 (TRIM37) has been demonstrated to function importantly during the progression of various cancers. However, the role of TRIM37 in gastric cancer (GC) remains elusive.

Materials and methods: TRIM37 mRNA and protein expressions were determined by qRT-PCR, Western blot, and immunohistochemical staining in GC specimens. The effects of TRIM37 on GC cells behavior were evaluated by transwell assays in vitro and metastasis assay in vivo, respectively. Besides, qRT-PCR, Western blot, and immunofluorescence staining were employed to detect the expressions of TRIM37 and epithelial-mesenchymal transition (EMT)-related markers.

Results: The present study revealed that TRIM37 mRNA or protein expression was significantly increased in GC tissues compared with that in paracancerous control tissues, and its aberrant overexpression was closely associated with clinical metastasis and poor prognosis in patients with GC. TRIM37 knockdown significantly suppressed GC cells migration and invasion in vitro, as well as metastasis in vivo. Inversely, TRIM37 overexpression exerted the opposite effects. Mechanistic studies suggested that SIP1-mediated EMT might be responsible for TRIM37facilitated GC cells migration and invasion.

Conclusion: Our findings revealed that high TRIM37 expression was associated with clinical metastasis and poor survival in patients with GC. TRIM37 promoted GC cells migration and invasion via EMT, mediated by the transcription factor SIP1, thus providing a candidate target for GC treatment.

Keywords: tripartite motif containing 37, gastric cancer, epithelial-mesenchymal transition

\section{Introduction}

Gastric cancer (GC) is one of the most common malignancies and the leading cause of cancer-related deaths worldwide, especially in People's Republic of China. ${ }^{1,2}$ Although considerable progress has been made in early diagnosis and treatment of GC in recent decades, including surgical techniques and chemotherapy, the prognosis of patients with GC remains poor due to the high probability of metastasis and recurrence. ${ }^{3,4}$ And the molecular mechanisms responsible for $\mathrm{GC}$ metastasis remain poorly characterized. Therefore, it is imperative to identify novel metastases-related gene targets and the underlying mechanisms driving GC metastasis, aiming at developing target-specific treatment for GC.

Recently, epithelial-mesenchymal transition (EMT) has emerged as a welldocumented molecular event that contributes to cancer cells invasion and metastasis. . $^{5,6}$ The idea that epithelial cells can downregulate epithelial properties and acquire 
mesenchymal features is commonly known as EMT to focus on its transient nature. During EMT, cells transform from an epithelial condition to a mesenchymal state, with the loss of cohesiveness and apical-basal polarity, a change of cell shape, a remodeling of the cytoskeleton, an increase in motility, and the acquisition of an invasive phenotype, which enables the upregulation of the epithelial marker E-cadherin and the downregulation of the mesenchymal marker N-cadherin. ${ }^{7}$ As already reported, this switch in cell differentiation and behavior could be controlled by transcription factors, including SIP1, Snail, Slug, ZEB1 and Twist. ${ }^{8}$ Given that mesenchymal-epithelial transition represents the reverse event, it deserves an intensive exploration of targeted genes-induced reversal of EMT in cancer metastasis.

Tripartite motif containing 37 (TRIM37), located in the 17q23 chromosomal region, contains RING finger region, a hallmark of E3 ubiquitin ligases, and belongs to the TRIM protein family, which functions importantly in cancer progression. ${ }^{9}$ In consideration of its E3 ligase activity in the ubiquitin-proteasome degradation system, TRIM37 has been demonstrated to result in extensive changes in gene expression, including multiple tumor suppressors in several types of cancers. ${ }^{9,10}$ Previous studies revealed that TRIM37 enhanced invasion and metastasis of cancer cells via EMT, including colorectal cancer, hepatocellular carcinoma (HCC), and glioma. ${ }^{11-13}$ However, to our knowledge, the expression pattern and the role of TRIM37 in GC progression have not yet been elucidated.

Therefore, the present study aimed to examine the clinical significances of TRIM37 expression in GC tissues and the role and the underlying mechanism of TRIM37 in the invasion and metastasis of GC.

\section{Materials and methods}

\section{Patients, specimens, and cell lines}

A total of $120 \mathrm{GC}$ tissue samples were obtained from surgical tissue blocks in the Fifth Affiliated Hospital of Nantong University. Corresponding adjacent normal gastric mucosas were used as control groups. None of these patients had received preoperative chemotherapy or radiotherapy. Among them, fresh tissues of 55 cases were used for the detection of TRIM37 mRNA and protein expressions. Besides, 120 cases were subjected to immunohistochemical stainings. This study was approved by the Ethics Committee of the Fifth Affiliated Hospital of Nantong University, and written informed consent was obtained from each patient.

The human GC cell lines (MKN45, MGC803, SGC7901, KATO-III, BGC823, SNU216, and AGS) and normal gastric epithelial GES-1 cells were obtained from the Type Culture Collection of the Chinese Academy of Sciences (Shanghai, People's Republic of China). KATO-III cells were cultured in $80 \%$ IMDM (ATCC, Manassas, VA, USA) supplemented with $20 \%$ FBS (Gibco, Waltham, MA, USA). The other cell lines were maintained in RPMI-1640 medium replenished with $10 \%$ FBS. Cells were incubated in a humidified atmosphere containing $5 \% \mathrm{CO}_{2}$ at $37^{\circ} \mathrm{C}$.

\section{RNA isolation and quantitative reverse transcription polymerase chain reaction (qRT-PCR)}

Total RNA was isolated from tissues and cells using Trizol reagent (Thermo Fisher Scientific, Waltham, MA, USA) and was converted into cDNA using the PrimeScript RT Reagent Kit (TaKaRa, Japan). The expression level of mRNA was determined by qRT-PCR using the SYBR Green assay kit (Takara, Japan). The relative mRNA level was calculated using the $2^{-\Delta \Delta \mathrm{Ct}}$ method. PCR primer sequences are shown in Table S1.

\section{Western blot analysis}

Western blot was conducted using standard procedures as described previously. ${ }^{14}$ Briefly, equal amounts of protein from tissues or cell lysates were separated on SDS-PAGE and then transferred to polyvinylidene fluoride membranes (EMD Millipore, Billerica, MA, USA). Membranes were incubated in 5\% defatted dry milk to diminish nonspecific binding, followed by incubation with a primary antibody at $4^{\circ} \mathrm{C}$ overnight. Afterward, membranes were incubated with a secondary antibody. The bands were detected by the enhanced chemiluminescence detection reagent (Thermo Fisher Scientifc, Waltham, MA, USA).

Primary antibody against TRIM37 was purchased from Abcam (Cambridge, UK). EMT-related antibodies (E-cadherin, SIP1, Snail, Slug, ZEB1, Twist, and N-cadherin) were purchased from CST (MA, USA). GAPDH antibody, used as the loading control, was bought from Bioworld Technology (MN, USA).

\section{Immunohistochemistry (IHC) and evaluation}

The approach for IHC was carried out as described previously. ${ }^{15}$ Briefly, after deparaffination, rehydration, antigen retrieval, and block, the slides were incubated overnight with the primary antibody TRIM37, followed by HRP-conjugated secondary antibody. Then, the slides were stained with DAB, counterstained with hematoxylin, and observed under the microscope by two independent pathologists. 
The staining intensity score was defined as follows: 0 = negative; 1 = weak intensity; 2 = moderate intensity; $3=$ strong intensity, and the staining area was scored as follows: $0=0 \%-5 \%, 1=6 \%-25 \%, 2=26 \%-50 \%, 3=$ more than $50 \%$. The final score was the sum of the intensity and extension scores. Scores of 0-2 were expected negative, and scores of 3-6 were expected positive.

\section{Lentivirus infection}

Commercially available TRIM37 short hairpin RNA (shRNA) and overexpression constructs were purchased from Genechem (Shanghai, People's Republic of China). Scrambled sequence or empty vector was used as a control. The sequence-specific shRNA to SIP1 was 5'-GAAGGAAGATTATGACACTATG-3'. Cell transfection was conducted using Lipofectamine 2000 following the manufacturer's protocols. The TRIM37 expression was determined by Western blot. The transfection efficiency was assessed by Western blot analysis.

\section{Immunofluorescence staining}

Following fixation with $4 \%$ paraformaldehyde and permeabilization with $0.2 \%$ Triton X-100, cells were blocked by $5 \%$ BSA. Subsequently, cells were incubated overnight at $4{ }^{\circ} \mathrm{C}$ with indicated antibodies against E-cadherin (CST) and N-cadherin (CST). After washes, cells were incubated with Alexa Fluor-conjugated secondary antibody (Bioworld Technology, St Louis Park, MN, USA) and counterstained with DAPI. Finally, cells were visualized and photographed using a fluorescence microscope.

\section{Cell migration and invasion assays}

Cell migration and invasion assays were conducted as described previously. ${ }^{16}$ Briefly, for cell migration assay, cells were seeded in serum-free medium and added to the top chamber ( $8 \mathrm{~mm}$ pore size; Corning Incorporated, Corning, NY, USA). For cell invasion assay, each chamber was coated additionally with Matrigel (BD Biosciences, San Jose, CA, USA). The medium with $20 \%$ FBS was seeded in the lower chamber as a chemoattractant. Following incubation for 24 hours, cells on the underside of each chamber were fixed by using $4 \%$ paraformaldehyde, stained with $0.5 \%$ crystal violet, and calculated in five random fields under a microscope.

\section{In vivo tumorigenesis}

To determine the effect of TRIM37 on tumor metastasis in vivo, cells were injected intravenously into the tail vein of 6-week-old male BALB/c nude mice. Four weeks after injection, the lungs of nude mice were harvested for the count of metastatic foci and subsequently processed for H\&E staining and Western blot analysis. The experimental procedures were approved by the Ethics Committee of the Fifth Affiliated Hospital of Nantong University and were carried out according to the institutional guidelines.

\section{Statistical analyses}

Chi-squared test was conducted to analyze clinicopathological characteristics of patients between different groups. Kaplan-Meier method and log-rank test were carried out to analyze the survival probabilities. All data were presented as the mean \pm SD and were analyzed by Student's $t$-test. All of the statistical analyses were performed using SPSS 21.0 software (IBM Corporation, Armonk, NY, USA). $P<0.05$ was considered statistically significant.

\section{Results}

TRIM37 expression is upregulated in GC tissues and correlated with the prognosis of patients with GC

To ascertain the function of TRIM37 in patients with GC, we first detected the expression levels of TRIM37 mRNA and protein in 55 pairs of GC tissues and paracancerous control tissues, respectively, by qRT-PCR and Western blot. As shown in Figure 1A and B, the qRT-PCR result revealed that the relative expression level of TRIM37 mRNA was significantly higher in tumor tissues compared with that in paracancerous control tissues. Similar results were observed in the TRIM37 protein analysis (Figure 1C). Furthermore, we performed the further verification with $120 \mathrm{GC}$ patient specimens through IHC assay (Figure 1D). The result indicated that TRIM37 expression was stably higher expressed in tumor tissues compared with that in paracancerous control tissues (Figure 1E), especially in metastatic tumor tissues (Figure 1F).

Next, a clinicopathological correlation study manifested that TRIM37 was significantly associated with $\mathrm{T}$ stage, pTNM stage, and lymph node metastasis, respectively $(P<0.001, P=0.001$, and $P<0.001)$ (Table 1), implying that TRIM37 might function importantly in cancer metastasis. More interestingly, the Kaplan-Meier curve demonstrated that positivity for TRIM37 was associated with shorter overall survival in patients with GC (Figure 1G).

\section{TRIM37 expression in GC cell lines}

The clinical data analysis demonstrated that TRIM37 overexpression was positively associated with tumor metastasis and unfavorable prognosis, which inspired us to investigate 
A

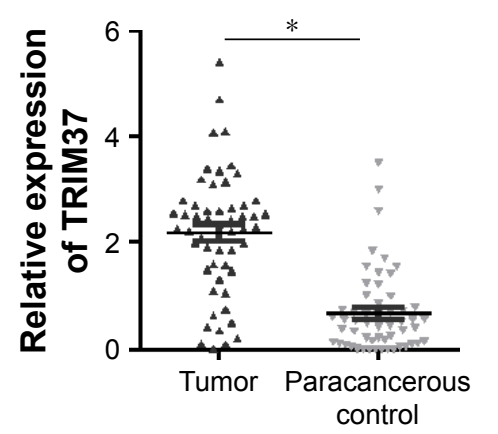

C
B

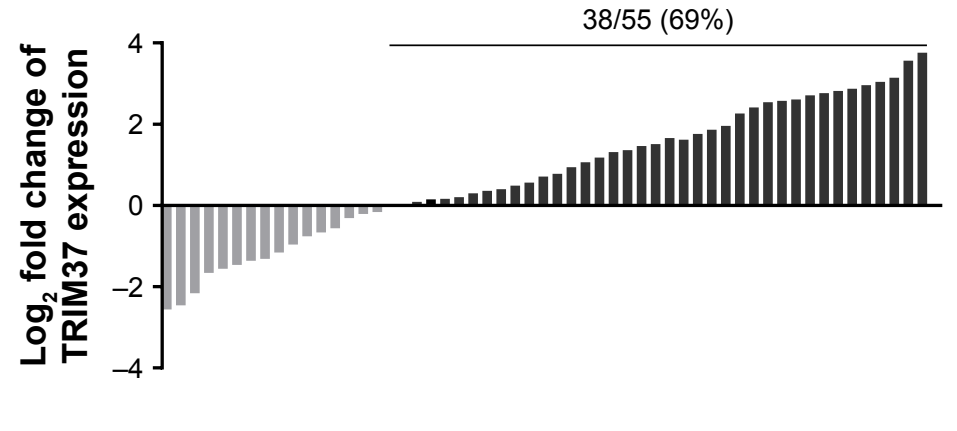

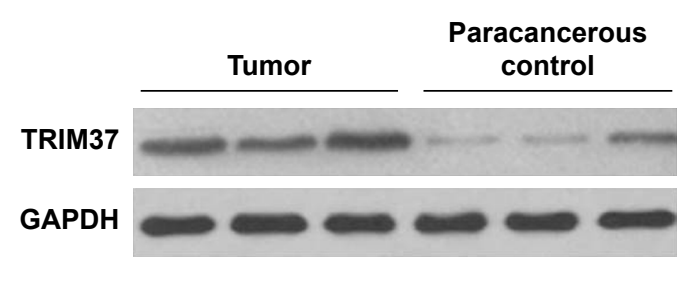

D

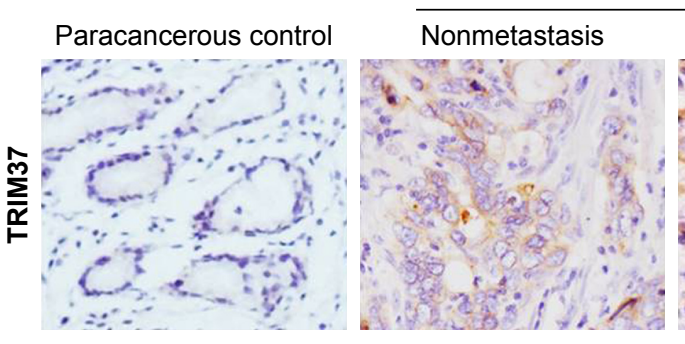

F

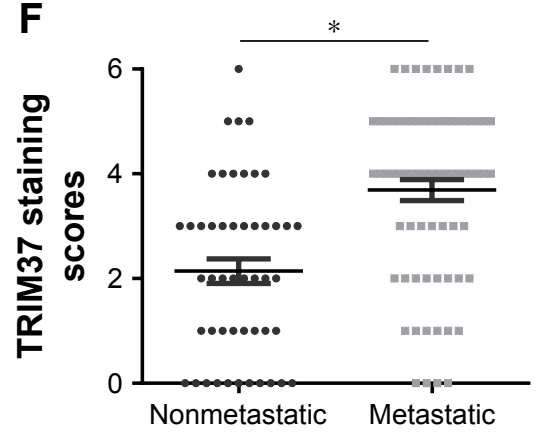

G

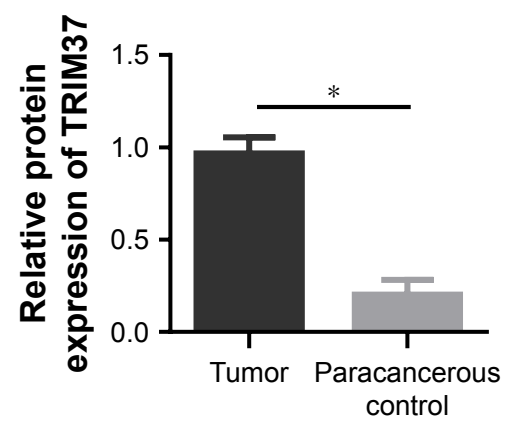

E

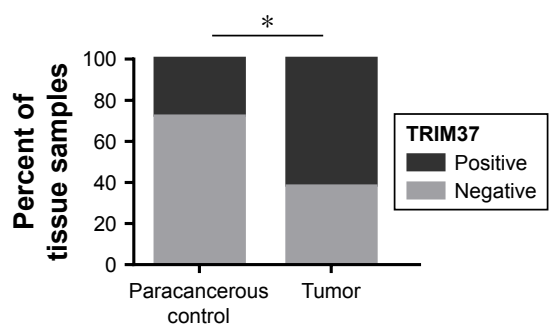

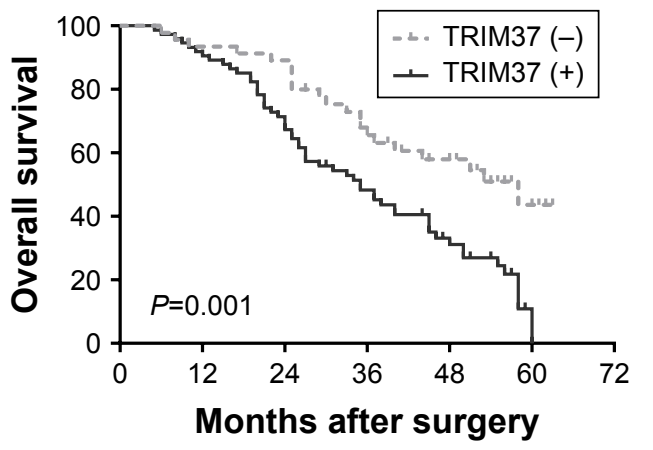

Figure I Relative TRIM37 expression in GC tissues and its clinical significances.

Notes: (A, B) qRT-PCR analysis of TRIM37 mRNA expression in GC tumor tissues and paracancerous control tissues. (C) Western blot analysis of TRIM37 protein expression in GC tumor tissues and paracancerous control tissues. (D) Representative IHC images of TRIM37 protein expression in clinical GC samples with or without metastasis and paracancerous control tissues. (E) Quantitative evaluation of TRIM37 protein expression in tumor tissues and paracancerous control tissues on the basis of staining scores. (F) Scatterplots of the average staining scores of TRIM37 expression in patients without or with metastasis. (G) Kaplan-Meier analysis of the correlation between TRIM37 expression and the overall survival of patients with GC. $* P<0.05$.

Abbreviations: GC, gastric cancer; IHC, immunohistochemistry; qRT-PCR, quantitative reverse transcription polymerase chain reaction; TRIM37, tripartite motif containing 37.

the functional role of TRIM37 in GC cell lines. We detected the endogenous expression of TRIM37 in six GC cell lines and normal gastric epithelial GES-1 cells by Western blot. Compared to GES-1 cells, TRIM37 expression was distinctly upregulated in GC cells (Figure 2A). Additionally, MKN45 and MGC803 cells with the highest TRIM37 expression were selected for transfection with shRNA lentivirus vector toward TRIM37 (Figure 2B), and KATO-III and AGS cells with the 
Table I Correlation with the clinicopathological features and TRIM37 expression in patients with GC

\begin{tabular}{|c|c|c|c|c|}
\hline \multirow[t]{2}{*}{ Features } & \multirow[t]{2}{*}{$\mathbf{n}$} & \multicolumn{2}{|l|}{ TRIM37 } & \multirow[t]{2}{*}{$P$-value } \\
\hline & & Negative & Positive & \\
\hline \multicolumn{5}{|l|}{ Age (years) } \\
\hline$\geq 60$ & 80 & 29 & 51 & \multirow[t]{2}{*}{0.507} \\
\hline$<60$ & 40 & 17 & 23 & \\
\hline \multicolumn{5}{|l|}{ Gender } \\
\hline Male & 79 & 34 & 45 & \multirow[t]{2}{*}{0.141} \\
\hline Female & 41 & 12 & 29 & \\
\hline \multicolumn{5}{|l|}{ Tumor size $(\mathrm{cm})$} \\
\hline$\geq 5$ & 69 & 22 & 47 & \multirow[t]{2}{*}{0.091} \\
\hline$<5$ & 51 & 24 & 27 & \\
\hline \multicolumn{5}{|c|}{ Lauren's classification } \\
\hline Diffuse & 30 & 9 & 21 & \multirow[t]{2}{*}{0.278} \\
\hline Intestinal & 90 & 37 & 53 & \\
\hline \multicolumn{5}{|c|}{ Lymphatic vessel invasion } \\
\hline With & 49 & 15 & 34 & \multirow[t]{2}{*}{0.148} \\
\hline Without & 71 & 31 & 40 & \\
\hline \multicolumn{5}{|l|}{ T stage } \\
\hline$T_{1}+T_{2}$ & 54 & 30 & 24 & \multirow[t]{2}{*}{$<0.00$ I $^{*}$} \\
\hline $\mathrm{T}_{3}+\mathrm{T}_{4}$ & 66 & 16 & 50 & \\
\hline \multicolumn{5}{|l|}{ pTNM stage } \\
\hline$I+I I$ & 51 & 28 & 23 & \multirow[t]{2}{*}{$0.00 I^{*}$} \\
\hline III + IV & 69 & 18 & 51 & \\
\hline \multicolumn{5}{|c|}{ Lymph node metastasis } \\
\hline $\begin{array}{l}\text { With } \\
\left(N_{1}+N_{2}+N_{3}\right)\end{array}$ & 71 & 14 & 57 & \multirow[t]{2}{*}{$<0.001 *$} \\
\hline Without $\left(\mathrm{N}_{0}\right)$ & 49 & 32 & 17 & \\
\hline
\end{tabular}

Note: *Mean $P<0.05$. Bold text represents T stage: $P=0.000$; $P$ TNM stage: $P=0.001$; and Lymph node metastasis: $P=0.000$.

Abbreviations: GC, gastric cancer; TRIM37, tripartite motif containing 37 .

lowest TRIM37 expression were selected for transfection with TRIM37-overexpression vector (Figure 2C).

\section{TRIM37 facilitates GC cells migration, invasion and metastasis in vitro and in vivo}

In the process of tumor metastasis, cancer cells tend to have stronger migration, invasion, and metastasis abilities. ${ }^{5}$ To characterize the effect of TRIM 37 on GC cells invasion and metastasis, we noticed that TRIM37 knockdown in MKN45 cells resulted in morphological alteration, from a more elongated and spindle-like mesenchymal shape to a rounded and cuboidal-like epithelial shape. Conversely, ectopic overexpressing of TRIM37 in AGS cells led to the opposite phenomenon (Figure 3A). Meanwhile, downregulation of TRIM37 expression suppressed MKN45 cells migration and invasion, whereas upregulation of TRIM37 expression accelerated AGS cells migration and invasion (Figure 3B). Together, these results indicated that TRIM37 promoted GC cells migration and invasion in vitro.

To further elucidate the role of TRIM37 in cells metastatic potential in vivo, the lung metastasis model was established via the injection of GC cells into the caudal vein in nude mice. The results revealed that TRIM37 knockdown in MKN45 cells hindered cells metastasis in vivo, as evidenced by a significantly less number of lung metastatic nodules (Figure 3C) and a conspicuous reversal of EMT (Figure 3D). As expected, TRIM37 overexpression had imposed remarkably opposite effects on AGS cells metastasis in vivo (Figure 3C and D). In summary, these results suggested that TRIM37 could promote GC cells metastasis in vivo.

\section{TRIM37 promotes GC cells invasion through SIPI-mediated EMT}

Accumulating evidences have established a vital role of aberrant EMT activation in many cancers metastasis process by endowing epithelial cells with their migratory and invasive attributes. ${ }^{5}$ In this study, we found that TRIM37 knockdown in MKN45 cells resulted in the upregulation of endogenous E-cadherin mRNA level and the downregulation of endogenous SIPI and N-cadherin mRNA levels. Whereas TRIM37 overexpression showed the opposite results in AGS cells. However, the mRNA levels of transcription factors (Snail, Slug, ZEB1, and Twist) were not observably affected in GC cells (Figure 4A). Besides Western blot analysis and immunofluorescence staining further demonstrated similar results (Figure 4B and C). Hence, we reasoned that TRIM37 might accelerate GC cells migration and invasion through EMT probably mediated by EMT-related transcription factor SIP1.

Next, to confirm the involvement of SIP1 in TRIM37mediated GC cells invasion and EMT, the SIP1 expression was silenced in GC cells (shNC/vector and transfected cells). We found that SIP1 silencing enhanced shTRIM37-mediated increase in E-cadherin and decrease in $\mathrm{N}$-cadherin (Figure 5A) and significantly potentiated shTRIM37-inhibited cell invasion (Figure 5B). Furthermore, shSIP1 led to a substantial reversal of TRIM37-induced EMT (Figure 5C) and partially reverted TRIM37-facilitated cell invasion (Figure 5D). Taken together, these data revealed that TRIM37 promoted GC cells invasion and EMT, at least in part, via the activation of transcriptional factor SIP1.

\section{Discussion}

Despite great achievements have been made in early diagnosis, surgical skills, and chemoradiotherapy over the past few decades, $\mathrm{GC}$ is still one of the most lethal malignancies 
A

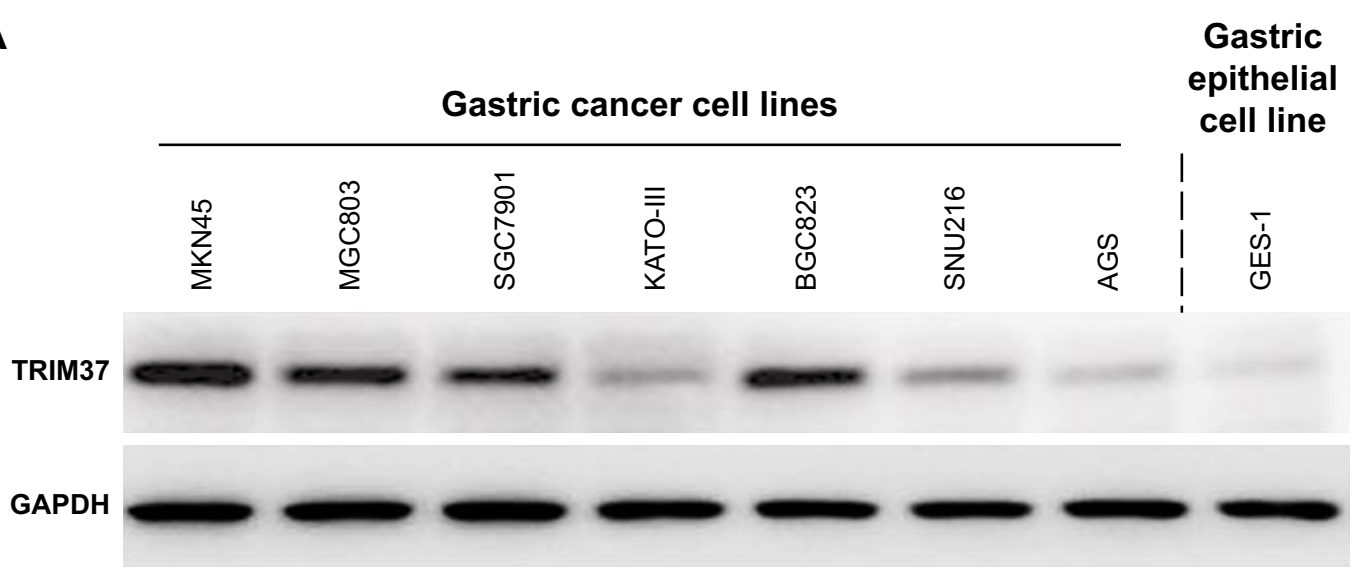

B
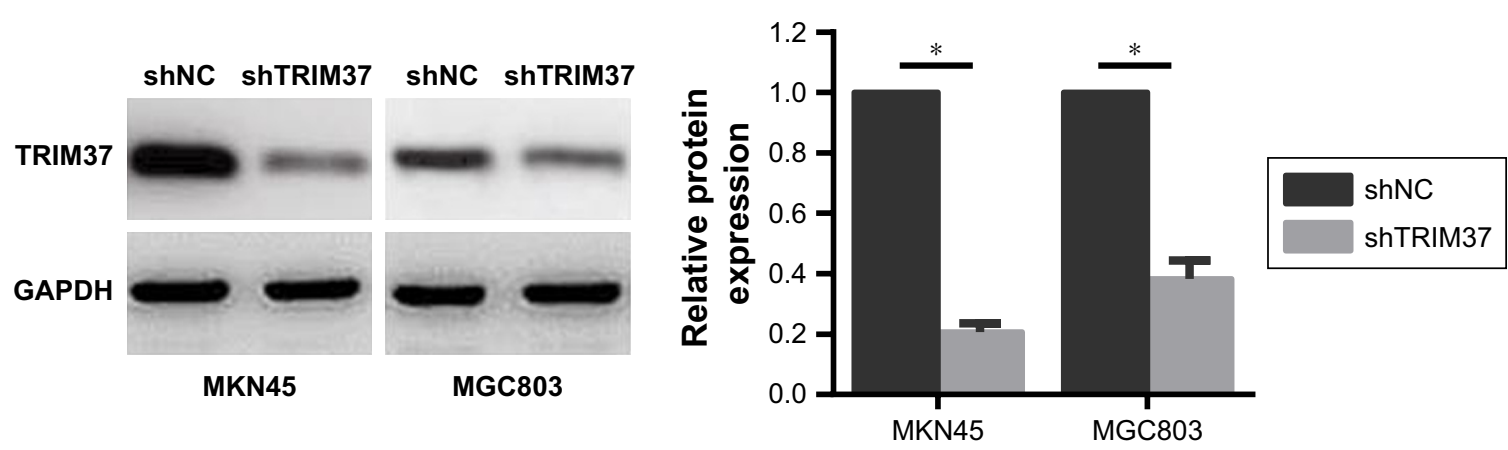

C

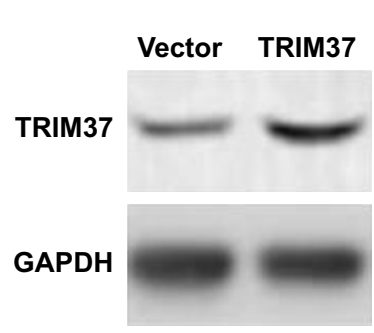

KATO-III

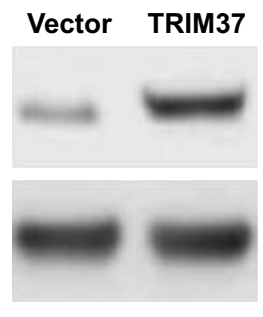

AGS

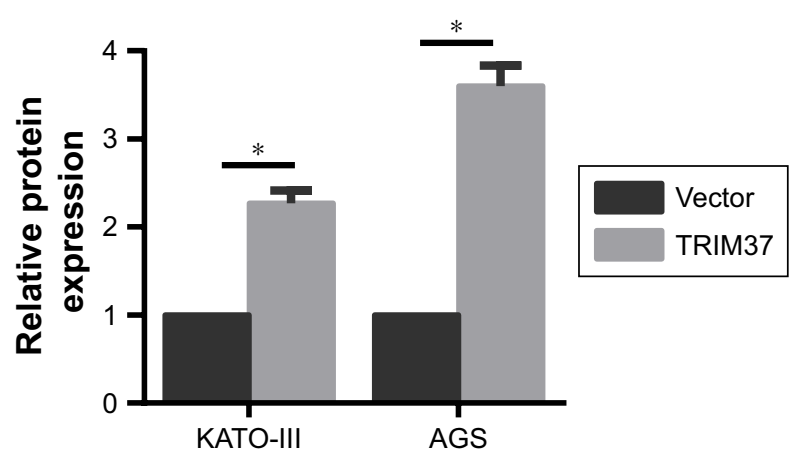

Figure 2 Relative TRIM37 protein expressions in GC cells.

Notes: (A) Western blot analysis of relative TRIM37 expression in GC cell lines and normal gastric epithelial GES-I cells. (B, C) Western blot analysis of TRIM37 expression levels in TRIM37-silencing cells (MKN45 and MGC803) and TRIM37-overexpressing cells (KATO-III and AGS). $* P<0.05$.

Abbreviations: GC, gastric cancer; TRIM37, tripartite motif containing 37.

worldwide, especially in People's Republic of China, where its morbidity and mortality have demonstrated a troubling health trend. ${ }^{1,17}$ Metastasis remains a dominating impediment to the curative treatment and bears a major responsibility for postsurgery recurrence and death in patients with $\mathrm{GC} .{ }^{18} \mathrm{Con}$ sidering that the spread of cancer cells to distant organs, from a relatively immobile type to a more invasive phenotype, is generally considered a key event during cancer progression and metastasis, novel effective strategies are urgently needed to restrain the metastatic dissemination of cancer cells. Consequently, the identification of novel therapeutic targets and a better understanding of the mechanisms involved in GC cells invasion and metastasis may be conducive to improving treatment efficacy.

Interestingly, mounting evidences demonstrated that TRIM37 was aberrantly upregulated in multiple types of cancer and identified as a key player influencing cancer cells migration, invasion, and metastasis. ${ }^{11-13}$ For example, a previous study has reported that TRIM37 was notably upregulated in human HCC tissues and was closely associated with clinical metastasis, and TRIM37 overexpression significantly enhanced the invasion and metastasis of HCC cells. ${ }^{12}$ In pancreatic cancer, TRIM37 was found to be noticeably higher in pancreatic cancerous tissues compared with that 
A

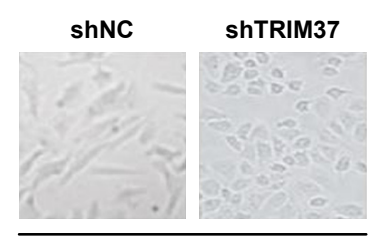

MKN45

B

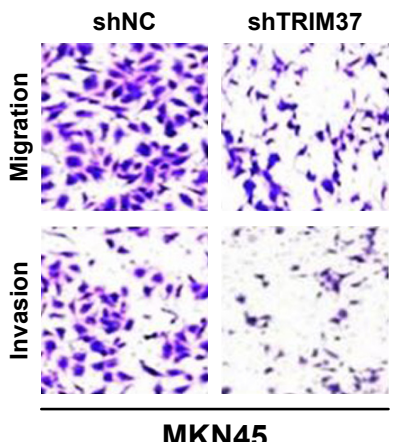

MKN45

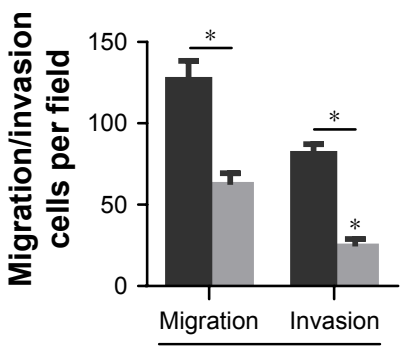

MKN45

shNC shTRIM37

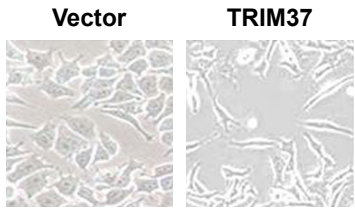

AGS
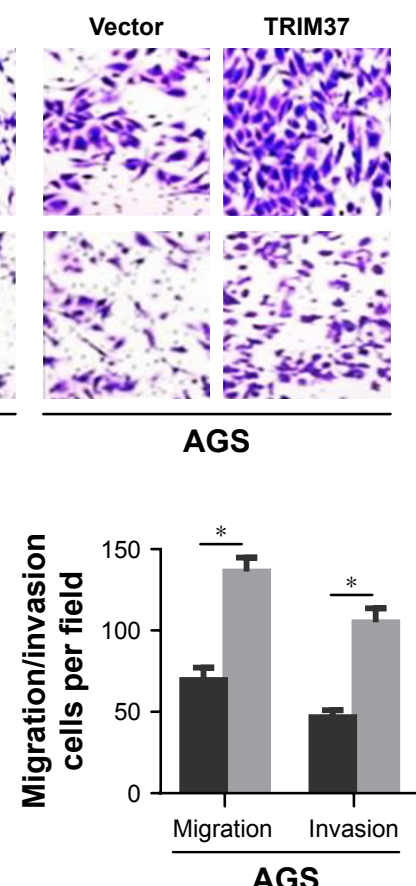

AGS

Vector TRIM37
C

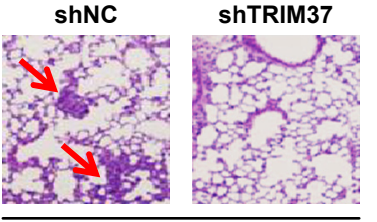

MKN45
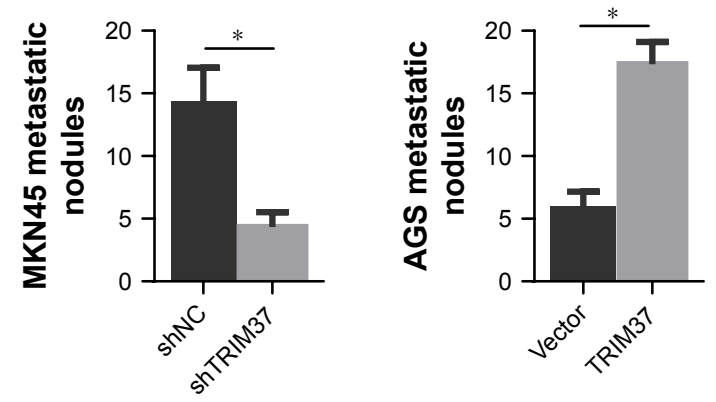

D

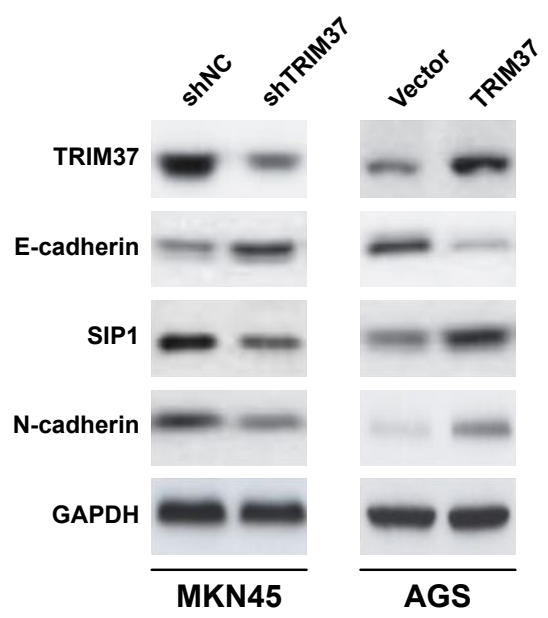

Figure 3 Effects of TRIM37 knockdown or overexpression on GC cells migration, invasion in vitro, and metastasis in vivo.

Notes: (A) Representative images of the effects of TRIM37 knockdown or overexpression on the morphology of GC cells. (B) The influences of TRIM37 silencing or overexpression on the migration and invasion abilities of GC cells were measured by transwell assay. (C) The effects of TRIM37 silencing or overexpression on GC cells metastasis in vivo. Metastatic tumor nodules (shown by red arrows). (D) Western blot analysis of expressions of TRIM37, E-cadherin, SIPI, and N-cadherin in lung metastatic nodules. *P $<0.05$. Abbreviations: GC, gastric cancer; TRIM37, tripartite motif containing 37.

in matched normal samples. Function analysis demonstrated that TRIM37 knockdown inhibited pancreatic cancer cells migration, while TRIM37 overexpression led to the opposite effect, suggesting the key role of TRIM37 in pancreatic cancer cells migration, and a potential target for pancreatic cancer treatment. ${ }^{19} \mathrm{Hu}$ and Gan also revealed that TRIM37 enhanced invasion and metastasis of colorectal cancer via EMT signaling. ${ }^{11}$ Collectively, these results inspired us to investigate the specific function of TRIM37 in GC invasion and metastasis and clarify its molecular mechanism. To test this idea, we first determined TRIM37 expression in tissues samples. The results demonstrated that TRIM37 expression was dramatically increased in GC samples compared with that in paracancerous control tissues and was closely correlated with advanced stage, which all suggested a poor prognosis. We next assessed whether TRIM37 could influence GC cells behavior. Our current study indicated that TRIM37 might promote the invasion and metastasis of GC cells via activating EMT signaling. Thus, it deserved a further analysis of the relationship between TRIM37 and EMT.

A well-known function of EMT is thought to act as a key contributor to cells invasion and metastasis in some cancers by the transition to a cell phenotype with a more spindle-like morphology, which reinforces the motility of individual cells and enables the development of an invasive phenotype. Hence, targeting EMT in cancer is increasingly accepted as a novel therapeutic strategy to prevent tumor dissemination in patients at high risk of developing metastatic lesions or to eradicate existing metastatic cancer 

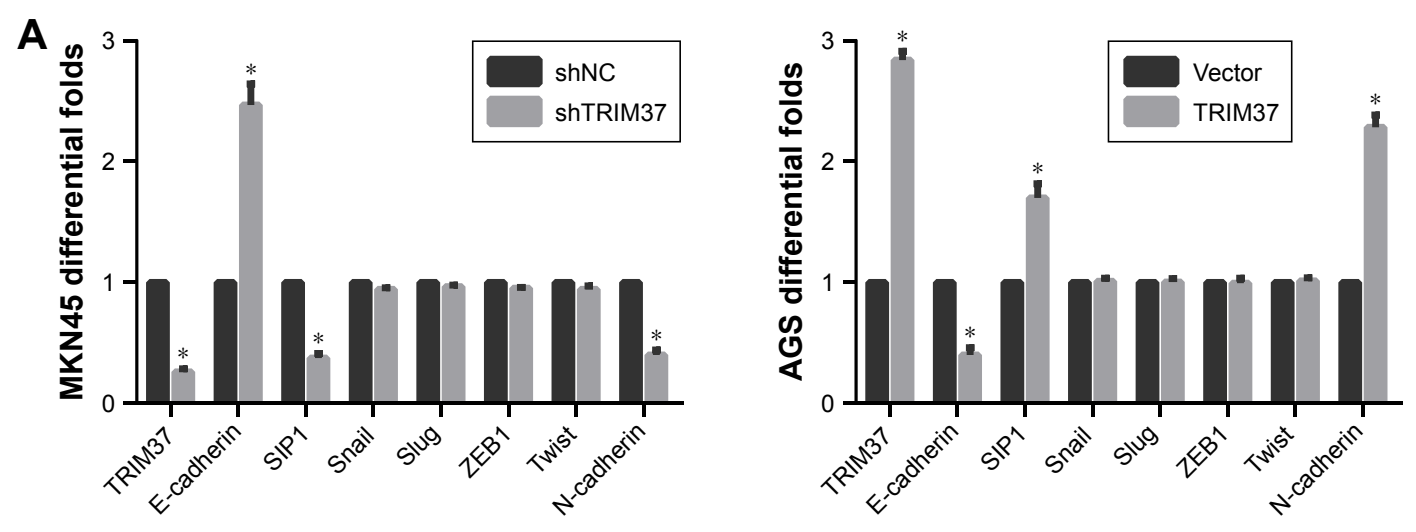

B
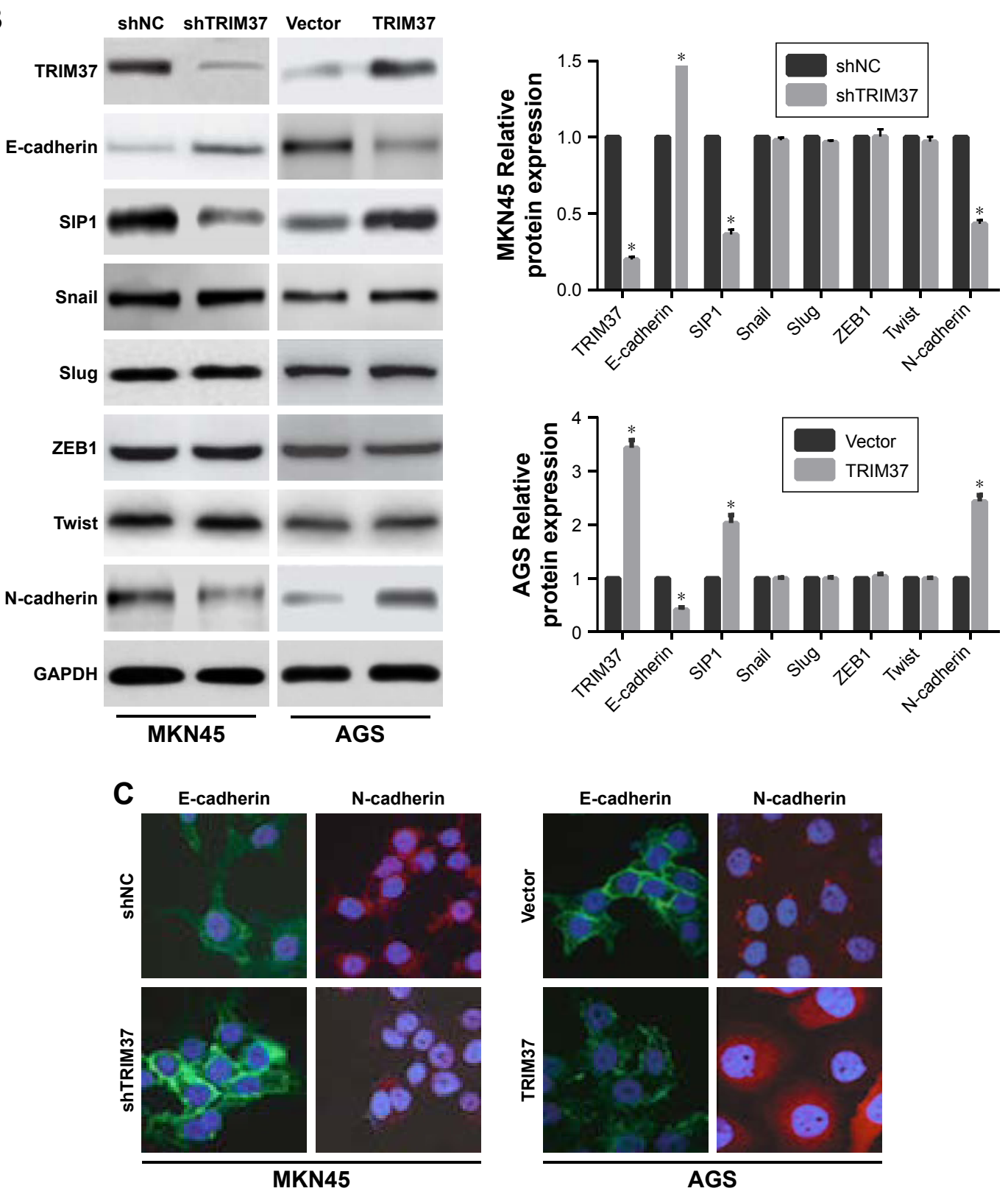

Figure 4 Effects of TRIM37 knockdown or overexpression on EMT-related markers in GC cells.

Notes: Following TRIM37 silencing or overexpression treatment, qRT-PCR (A), Western blot (B), and immunofluorescence (C) were employed to detect the expressions of EMT-related markers in TRIM37-altered cells, including E-cadherin, N-cadherin, and transcription factors (SIPI, Snail, Slug, ZEBI, and Twist). *P $<0.05$.

Abbreviations: EMT, epithelial-mesenchymal transition; GC, gastric cancer; qRT-PCR, quantitative reverse transcription polymerase chain reaction; TRIM37, tripartite motif containing 37. 
A
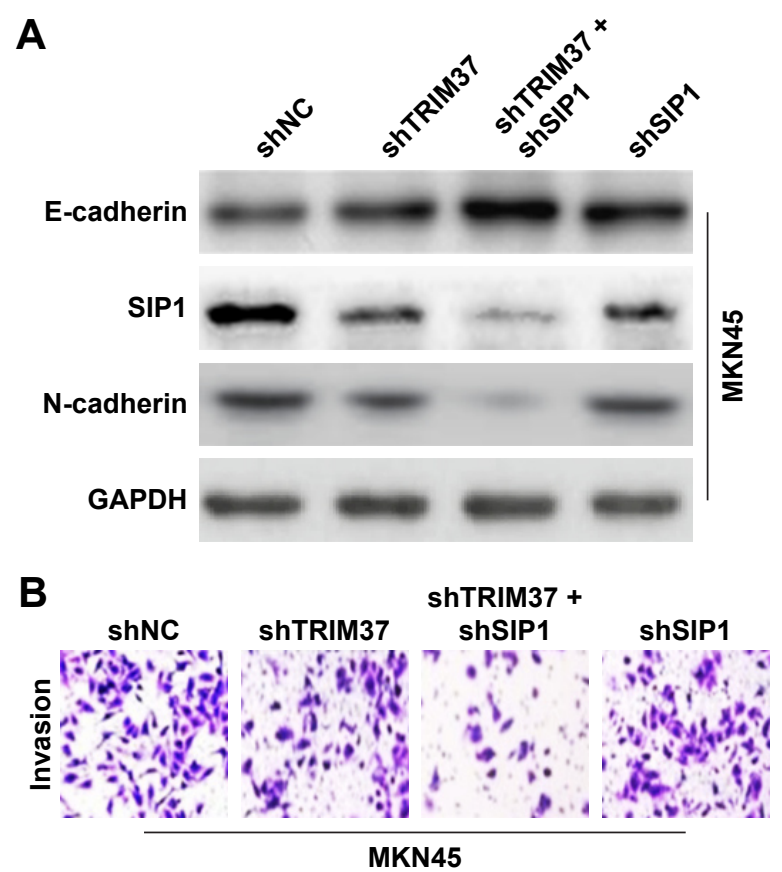

MKN45

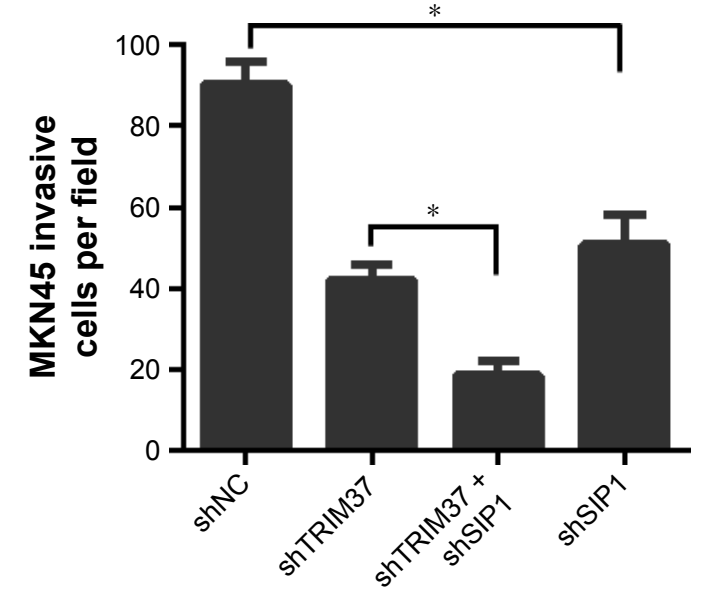

C
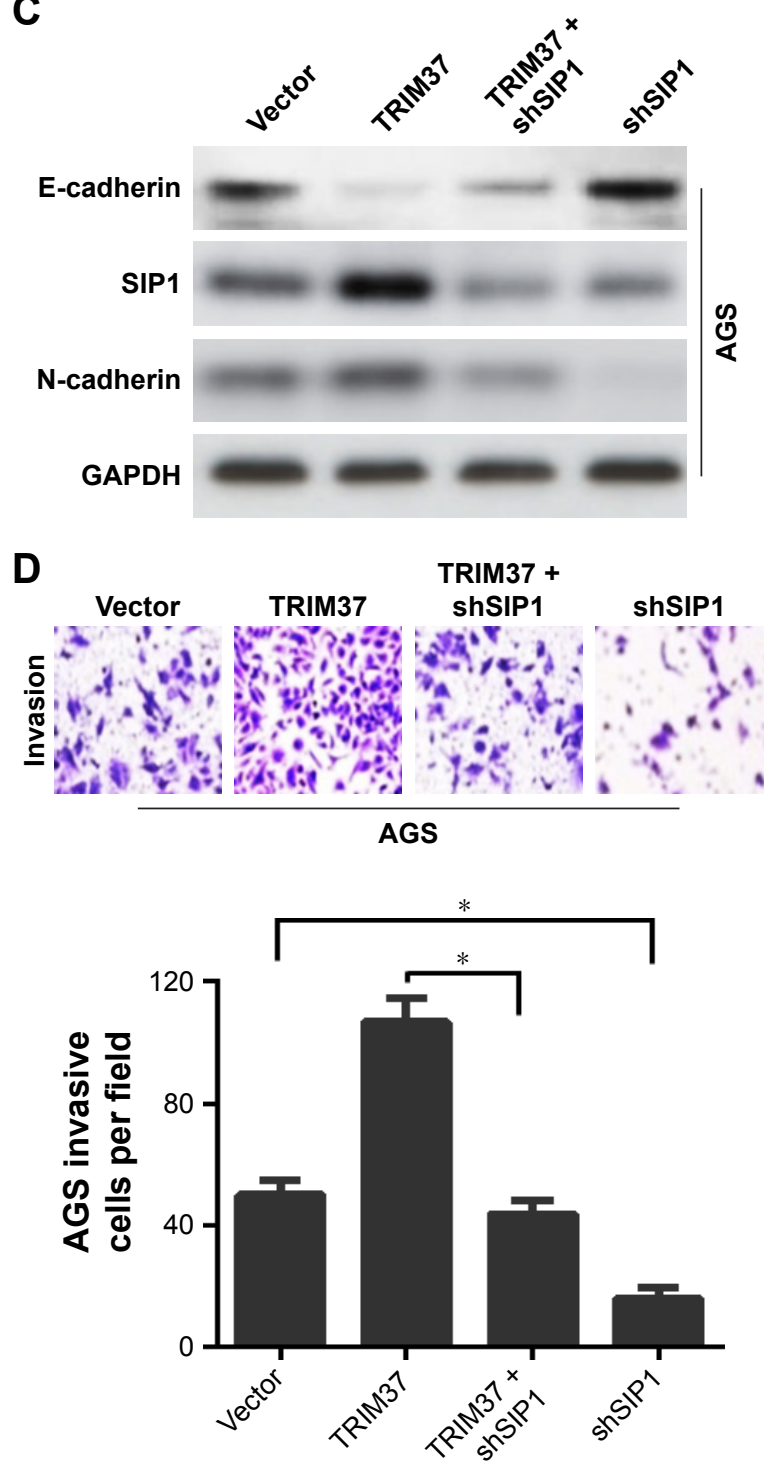

Figure 5 SIPI-dependent mechanism of TRIM37-facilitated GC cells EMT and invasion.

Notes: (A, C) Western blot analysis of the SIPI-dependent mechanism of TRIM37-inducing GC cells EMT. (B, D) Confirmation of the SIPI-dependent mechanism of TRIM37-inducing GC cells invasion by transwell assay. $* P<0.05$.

Abbreviations: EMT, epithelial-mesenchymal transition; GC, gastric cancer; TRIM37, tripartite motif containing 37.

cells in patients with more advanced stage.$^{20}$ Noticeably, substantial efforts have been made to screen novel targeted genes that accelerate tumor cell evolution via activating EMT signaling..$^{21,22}$

Here, we found that TRIM37 overexpression promoted the EMT process together with the decreased expression of E-cadherin and increased expression of $\mathrm{N}$-cadherin, whereas TRIM37 knockdown achieved a reversal of EMT with the suppression of GC cells invasion and metastasis. Additionally, extensive evidence has confirmed that transcription factor (such as SIP1, Snail, Slug, ZEB1, and Twist), as a regulator of EMT, was activated in many types of cancer, thus facilitating cells invasion and metastasis. ${ }^{23}$ In this study, the results showed that TRIM37 overexpression led to the upregulated expression of SIP1 and in turn accelerated EMT progression, thereby strengthening the migration and invasion abilities of GC cells. Inversely, TRIM37 silencing resulted in the opposite effects. To validate that SIP1 was involved in TRIM37-induced EMT, the endogenous SIP1 expression was reduced in GC cells, which weakened TRIM37-induced EMT and cell invasion and enhanced shTRIM37-inhibited EMT and cell invasion. Therefore, we could reasonably assume that TRIM37 promoted GC cells invasion and metastasis by regulating SIP1-mediated EMT.

However, the mechanism of SIP1 regulating EMT in this study remains elusive. Transcription factors have 
distinguishing expression profiles; their contributions to EMT depend on the cell or tissue type involved and the signaling pathways that drive EMT. They often regulate the expression of each other and functionally cooperate at target genes, and additional transcription factors further define the EMT transcription program and initiate EMT progression. Taken together, transcription factors coordinate the repression of epithelial genes and the induction of mesenchymal genes, and often the same transcription factors direct both repression and activation. ${ }^{5}$ Although the further exploration of the direct or indirect relationship between SIP1 and EMT is very complex, this deserves an in-depth study in the following research.

\section{Conclusion}

In summary, this present study revealed that TRIM37 was overexpressed in GC tissues and was significantly associated with clinical metastasis and an unfavorable prognosis of patients with GC. Besides TRIM37 could promote GC cells invasion and metastasis via activation of SIP1-indcued EMT, thus possibly providing a potential specific target for patients with GC.

\section{Acknowledgments}

This work was supported in part by funding from Taizhou Science and Technology Support Plan (Social Development) Project (TS201732) and Medical Science and Technology Development Foundation of Jiangsu University (JLY20160148 and JLY20160149).

\section{Disclosure}

The authors report no conflicts of interest in this work.

\section{References}

1. Siegel RL, Miller KD, Jemal A. Cancer statistics, 2018. CA Cancer J Clin. 2018;68(1):7-30.

2. Chen W, Zheng R, Baade PD, et al. Cancer statistics in China, 2015. CA Cancer J Clin. 2016;66(2):115-132.

3. Xu W, Yang Z, Lu N. Molecular targeted therapy for the treatment of gastric cancer. J Exp Clin Cancer Res. 2016;35:1.

4. Catalano V, Labianca R, Beretta GD, Gatta G, de Braud F, Van Cutsem E. Gastric cancer. Crit Rev Oncol Hematol. 2009;71(2):127-164.

5. Lamouille S, Xu J, Derynck R. Molecular mechanisms of epithelialmesenchymal transition. Nat Rev Mol Cell Biol. 2014;15(3):178-196.
6. He SJ, Xiang CQ, Zhang Y, LuXT, Chen HW, Xiong LX. Recent progress on the effects of microRNAs and natural products on tumor epithelialmesenchymal transition. Onco Targets Ther. 2017;10:3435-3451.

7. Acloque H, Adams MS, Fishwick K, Bronner-Fraser M, Nieto MA. Epithelial-mesenchymal transitions: the importance of changing cell state in development and disease. J Clin Invest. 2009;119(6):1438-1449.

8. Singh M, Yelle N, Venugopal C, Singh SK. EMT: mechanisms and therapeutic implications. Pharmacol Ther. 2018;182:80-94.

9. Bhatnagar S, Gazin C, Chamberlain L, et al. TRIM37 is a new histone H2A ubiquitin ligase and breast cancer oncoprotein. Nature. 2014; 516(7529):116-120.

10. Kallijärvi J, Lahtinen U, Hämäläinen R, Lipsanen-Nyman M, Palvimo JJ, Lehesjoki AE. TRIM37 defective in mulibrey nanism is a novel RING finger ubiquitin E3 ligase. Exp Cell Res. 2005;308(1):146-155.

11. Hu CE, Gan J. TRIM37 promotes epithelial-mesenchymal transition in colorectal cancer. Mol Med Rep. 2017;15(3):1057-1062.

12. Jiang J, Yu C, Chen M, Tian S, Sun C. Over-expression of TRIM37 promotes cell migration and metastasis in hepatocellular carcinoma by activating Wnt/B-catenin signaling. Biochem Biophys Res Commun. 2015;464(4):1120-1127.

13. Tang SL, Gao YL, Wen-Zhong H. Knockdown of TRIM37 suppresses the proliferation, migration and invasion of glioma cells through the inactivation of PI3K/Akt signaling pathway. Biomed Pharmacother. 2018;99:59-64.

14. Chen D, Zhou H, Liu G, Zhao Y, Cao G, Liu Q. SPOCK1 promotes the invasion and metastasis of gastric cancer through Slug-induced epithelialmesenchymal transition. J Cell Mol Med. 2018;22(2):797-807.

15. Chen D, Cao G, Qiao C, Liu G, Zhou H, Liu Q. Alpha B-crystallin promotes the invasion and metastasis of gastric cancer via NF- $\mathrm{KB}-$ induced epithelial-mesenchymal transition. J Cell Mol Med. 2018; 22(6):3215-3222.

16. Chen D, Liu G, Xu N, et al. Knockdown of ARK5 expression suppresses invasion and metastasis of gastric cancer. Cell Physiol Biochem. 2017;42(3):1025-1036.

17. Xu AM, Huang L, Liu W, Gao S, Han WX, Wei ZJ. Neoadjuvant chemotherapy followed by surgery versus surgery alone for gastric carcinoma: systematic review and meta-analysis of randomized controlled trials. PLoS One. 2014;9(1):e86941.

18. Chaffer CL, Weinberg RA. A perspective on cancer cell metastasis. Science. 2011;331(6024):1559-1564.

19. Jiang J, Tian S, Yu C, Chen M, Sun C. TRIM37 promoted the growth and migration of the pancreatic cancer cells. Tumour Biol. 2016;37(2): 2629-2634.

20. Davis FM, Stewart TA, Thompson EW, Monteith GR. Targeting EMT in cancer: opportunities for pharmacological intervention. Trends Pharmacol Sci. 2014;35(9):479-488.

21. Li J, Zhen L, Zhang Y, et al. Circ-104916 is downregulated in gastric cancer and suppresses migration and invasion of gastric cancer cells. Onco Targets Ther. 2017;10:3521-3529.

22. Sui X, Zhou H, Zhu L, Wang D, Fan S, Zhao W. CUL4A promotes proliferation and metastasis of colorectal cancer cells by regulating $\mathrm{H} 3 \mathrm{~K} 4$ trimethylation in epithelial-mesenchymal transition. Onco Targets Ther. 2017;10:735-743.

23. Goossens S, Vandamme N, van Vlierberghe P, Berx G. EMT transcription factors in cancer development re-evaluated: beyond EMT and MET. Biochim Biophys Acta Rev Cancer. 2017;1868(2):584-591. 


\section{Supplementary material}

Table SI Primers designed for $\mathrm{qRT}$-PCR

\begin{tabular}{l|l|l}
\hline \multirow{2}{*}{ Genes } & Sequence $\left(\mathbf{5}^{\prime} \mathbf{- 3}^{\prime} \mathbf{)}\right.$ \\
\cline { 2 - 3 } & Sense & Antisense \\
\hline TRIM37 & TCAGCTGTATTAGGCGCTGG & ACTTCTTCTGCCCAACGACA \\
SIPI & CGAGAGCTACACGTTCACGG & GGGTGTCGAGGGAAAAATAGG \\
Snail & CAAGAGGCGCAAACAAGCC & GGTTGGCAATACCGTCATCC \\
Slug & AAGGCCTTCTCTAGGCCCT & CGCAGGTTGGAGCGGTCAG \\
ZEBI & TTCGGACCCACACATTACCT & GCAGTGAGGGCAAGAAAAAG \\
Twist & GATGATGAATGCGAGTCAGATGC & ACAGCAGTGTCTTGTTGTTGT \\
N-cadherin & CAGCTACGCCTTCTCGGTCT & CTGTCCATTTTCTCCTTCTCTGGA \\
GAPDH & TCAGGCGTCTGTAGAGGCTT & ATGCACATCCTTCGATAAGACTG \\
\hline
\end{tabular}

Abbreviation: $\mathrm{RRT}-\mathrm{PCR}$, quantitative reverse transcription polymerase chain reaction.

\section{Publish your work in this journal}

OncoTargets and Therapy is an international, peer-reviewed, open access journal focusing on the pathological basis of all cancers, potential targets for therapy and treatment protocols employed to improve the management of cancer patients. The journal also focuses on the impact of management programs and new therapeutic agents and protocols on
Dovepress

patient perspectives such as quality of life, adherence and satisfaction. The manuscript management system is completely online and includes a very quick and fair peer-review system, which is all easy to use. Visit http://www.dovepress.com/testimonials.php to read real quotes from published authors.

Submit your manuscript here: http://www.dovepress.com/oncotargets-and-therapy-journal 